importance of increasing education and awareness, training, and the use of specialized equipment in order to minimize noise exposure. Regulating onboard noise levels is necessary to avoid noise-related health problems.

\section{P-305 A MEDIA SURVEILLANCE ANALYSIS OF COVID-19 WORKPLACE OUTBREAKS IN CANADA AND THE UNITED STATES}

'Shelby Fenton, Cheryl Peters, Emma Quinn, Ela Rydz, Hugh Davies, Robert Macpherson, Chris McLeod, Mieke Koehoorn, Emily Heer. ${ }^{\top}$ Alberta Health Services, Canada

\subsection{6/OEM-2021-EPI.267}

Introduction The news media is one of the most accessible sources of information regarding COVID-19 transmission in the workplace in the absence of other occupational data. Only a few public health agencies in Canada and the United States have publically reported detailed occupation information for non-health care worker COVID-19 cases.

Objective We conducted a media surveillance analysis to identify new or emerging occupational groups at risk of exposure to the SARS-CoV-2 virus ('COVID-19 exposure').

Methods We searched the Factiva database for media articles reporting COVID-19 workplace outbreaks (February 1 December 22, 2020). Job titles were coded to the 2016 National Occupational Classification (V1.3) and industries to the 2017 North American Industry Classification System (V3.0). Occupations with COVID-19 workplace transmission identified in media articles were compared and contrasted with the same occupation in the Vancouver School of Economics (VSE) COVID Risk Tool by risk rating (seven categories between very high to very low).

Results We identified 1,111 unique COVID-19 workplace outbreaks in the media. After nurse aides, orderlies and patient service associates, industrial butchers and meat cutters, poultry preparers and related workers had the most workplace outbreaks reported in the media $(n=79)$ but were rated as medium risk occupations for COVID-19 transmission in the VSE COVID Risk Tool. Outbreaks were also reported in the media among material handlers $(n=61)$ and general farm workers $(n=28)$ but were rated medium-low risk and low risk, respectively. Outbreaks reported in the media among food and beverage services $(n=72)$ and cashiers $(n=60)$ were identified as high risk occupations in the VSE COVID Risk Tool.

Conclusion Media surveillance can identify COVID-19 workplace outbreaks and indicate transmission risk. Our results point to key determinants of health that compound the risk of COVID-19 exposure in the workplace, and highlight the importance of collecting occupation data during a pandemic.

\section{P-309 INVESTIGATING HEALTH AND OTHER CHARACTERISTICS OF MILITARY VETERANS AUTHORIZED TO RECEIVE MEDICINAL CANNABIS IN CANADA}

${ }^{1}$ Angela Czarina Mejia, Mieke Koehoorn, Hugh Davies, Amy Hall, Linda VanTil. 'University of British Columbia - Vancouver, Canada

10.1136/OEM-2021-EPI.268
Introduction Veterans Affairs Canada (VAC) has reimbursed cannabis for medical purposes (CMP) since 2008. However, to date little is known about the characteristics of Veterans authorized for CMP, and whether these differ by authorization amount.

Objectives To descriptively summarize social, health, and other characteristics of Canadian Regular Force Veterans authorized to receive CMP from Veterans Affairs Canada.

Methods A linked database of CMP authorizations was developed using VAC reimbursement files, VAC client records, and military personnel data. Analyses were limited to 13,173 Regular Force Veterans residing in Canada with an active authorization as of December 31, 2020. CMP authorization amounts (mean and categorical) were summarized by sociodemographic factors, pensionable conditions and benefits, and military service characteristics.

Results Overall, the average amount of a CMP authorization among Canadian Veterans was 3.6 grams/day. For sociodemographic characteristics, the highest average amounts were observed among Veterans who were aged 30 to 39 years $(4.2 \mathrm{~g} /$ day $)$, male $(3.7 \mathrm{~g} / \mathrm{day})$, separated/divorced/widowed $(3.8 \mathrm{~g} /$ day), and residing in the provinces of New Brunswick $(4.7 \mathrm{~g} /$ day), Newfoundland and Labrador (4.1g/day) and Manitoba (4.1g/day). For conditions documented as part of the VAC benefit process, the highest average amounts were observed among Veterans with mental health $(3.9 \mathrm{~g} /$ day) and hearing loss conditions (3.7g/day). For military service characteristics (sub-sample of 9,200 Veterans) the highest average amounts were observed among Veterans with a more recent release year, peaking in 2016 (4.2g/day); and among those who were Junior Non-Commissioned Members ( $4.0 \mathrm{~g} /$ day), had served in the army $(4.0 \mathrm{~g} / \mathrm{day})$, and released from the military involuntarily $(4.8 \mathrm{~g} /$ day $)$.

Conclusion This descriptive epidemiology provides new insights on the characteristics of a large population of veterans with medical cannabis authorizations in Canada. This will be used to inform further research on associations between CMP authorizations and wellness outcomes among military veterans.

\section{P-314 ESTABLISHING A EUROPEAN-AMERICAN POOLED COHORT OF STYRENE EXPOSED REINFORCED PLASTICS WORKERS}

${ }^{1}$ Mette Christensen, Damien McElvenny, Yvette Christopher-de Vries, loannis Basinas, Martie van Tongeren, John Cherrie, Georgia Ntani, Vanessa Cox, David Coggon, Stephen Bertke, Robert Daniels, Nicola Caranci, Stefano Mattioli, Stefania Curti, Eero Pukkala. 'Aarhus University, Denmark

\subsection{6/OEM-2021-EPI.269}

Introduction Styrene is genotoxic, an animal carcinogen, associated with lymphohematopoietic malignancies in humans, and classified as a group $2 \mathrm{~A}$ carcinogen by the IARC.

Objective To combine and harmonize existing cohorts of reinforced plastics industry workers with the aim to study exposure-response relations for styrene and subtypes of lymphohematopoietic and other malignancies as well as nonmalignant diseases.

Methods Six European cohorts included in an earlier IARC coordinated cohort and one US cohort participated. They all have been previously used in investigation of the health risks of styrene exposure. They will be updated with extended follow-up until 2019-2020 in national registries for mortality 